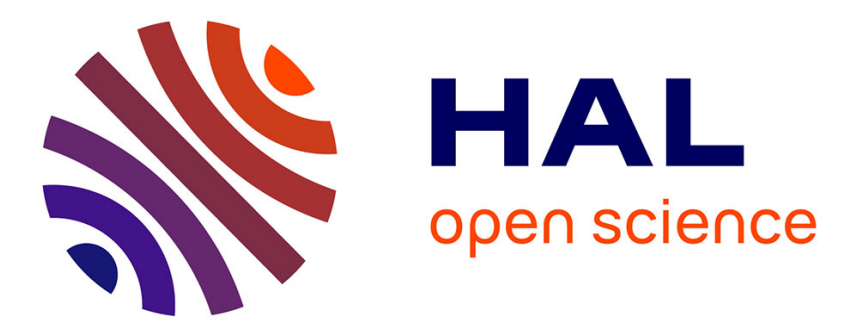

\title{
Thermal properties study of silicon nanostructures by photoacoustic techniques
}

K Dubyk, T Nychyporuk, V Lysenko, Konstantinos Termentzidis, Guillaume Castanet, F Lemoine, D. Lacroix, Mykola Isaiev

\section{- To cite this version:}

K Dubyk, T Nychyporuk, V Lysenko, Konstantinos Termentzidis, Guillaume Castanet, et al.. Thermal properties study of silicon nanostructures by photoacoustic techniques. Journal of Applied Physics, 2020, 127 (22), pp.225101. 10.1063/5.0007559 . hal-03418227v2

\section{HAL Id: hal-03418227 \\ https://hal.science/hal-03418227v2}

Submitted on 6 Nov 2021

HAL is a multi-disciplinary open access archive for the deposit and dissemination of scientific research documents, whether they are published or not. The documents may come from teaching and research institutions in France or abroad, or from public or private research centers.
L'archive ouverte pluridisciplinaire HAL, est destinée au dépôt et à la diffusion de documents scientifiques de niveau recherche, publiés ou non, émanant des établissements d'enseignement et de recherche français ou étrangers, des laboratoires publics ou privés. 


\section{Thermal properties study of silicon nanostructures by photoacoustic techniques}

Cite as: J. Appl. Phys. 127, 225101 (2020); https://doi.org/10.1063/5.0007559

Submitted: 17 March 2020 . Accepted: 25 May 2020 . Published Online: 08 June 2020

K. Dubyk (D), T. Nychyporuk, V. Lysenko, K. Termentzidis (D), C. Castanet (D), F. Lemoine, D. Lacroix (D), and M. Isaiev (iD)

\section{Lock-in Amplifiers up to $600 \mathrm{MHz}$}
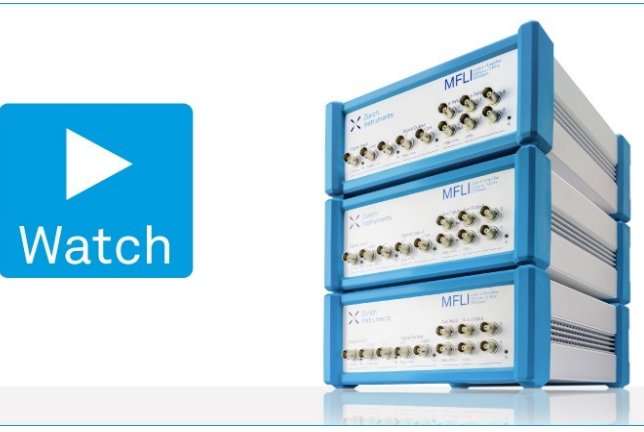


\title{
Thermal properties study of silicon nanostructures by photoacoustic techniques
}

Cite as: J. Appl. Phys. 127, 225101 (2020); doi: 10.1063/5.0007559

Submitted: 17 March 2020 . Accepted: 25 May 2020 .

Published Online: 8 June 2020

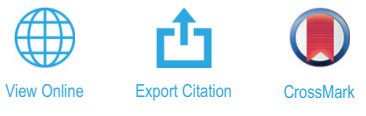

K. Dubyk, ${ }^{7, a)}$ (i) T. Nychyporuk, ${ }^{2}$ V. Lysenko, ${ }^{3,4}$ K. Termentzidis, ${ }^{5}$ (i) G. Castanet, ${ }^{6}$ (D) F. Lemoine, ${ }^{6}$ D. Lacroix, ${ }^{6}$ (D) and M. Isaiev ${ }^{6}$ (i)

\author{
AFFILIATIONS \\ ${ }^{1}$ Taras Shevchenko National University of Kyiv, 64/13, Volodymyrska Str., 01601 Kyiv, Ukraine \\ ${ }^{2}$ Université de Lyon, Institut des Nanotechnologies de Lyon INL-UMR5270, CNRS, INSA de Lyon, Villeurbanne F-69621, France \\ ${ }^{3}$ Nanotechnology Institute of Lyon (INL), UMR CNRS 5270, University of Lyon, 69361 Lyon, France \\ 4Light Matter Institute, UMR-5306, Claude Bernard University of Lyon/CNRS, Université de Lyon, 69622 Villeurbanne Cedex, France \\ ${ }^{5}$ Université de Lyon, CNRS, CETHIL, Villeurbanne F-69000, France \\ ${ }^{6}$ Université de Lorraine, CNRS, LEMTA, Nancy F-54000, France
}

${ }^{a)}$ Author to whom correspondence should be addressed: kateryna.dubyk@gmail.com

\begin{abstract}
The photoacoustic method with piezoelectric detection for the simultaneous evaluation of the thermophysical properties is proposed. The approach is based on the settling of an additional heat sink for redistribution of heat fluxes deposited on the sample surface. First, the approach was tested on the porous silicon with well-defined morphology and well-studied properties. Then, heat capacity and thermal conductivity of silicon nanowire arrays were investigated by recovering the experimental data through numerical simulations. The decrease in heat capacity and effective thermal conductivity of the samples upon increasing thickness and porosity of the sample was observed. Such a behavior could be caused by the increase of the structure heterogeneity. In particular, this can be related to a larger disorder (increased density of broken nanowires and larger porosity) that appears during the etching process of the thick layers.
\end{abstract}

Published under license by AIP Publishing. https://doi.org/10.1063/5.0007559

\section{INTRODUCTION}

Silicon-based materials are "sine qua non" in micro/nano/ opto-electronics. ${ }^{1-5}$ The continuous size reduction of such devices and the growing aspect of their efficient integration naturally lead to improve thermal management. Therefore, understanding heat transfer phenomena at the nano- and micro-scale is crucial for performance stability and reliability of nano/micro-devices.

Numerous in-contact and out-of-contact experimental methods are used in the literature for thermal transport property studies. With in-contact configurations, the sample is directly heated with a source that can be either a microprobe as in scanning thermal microscopy, ${ }^{6,7}$ a thin-film electrical resistor deposited onto the surface of the sample as in the $3 \omega$-method, ${ }^{8,9}$ a bolometer $^{10}$ as in pulse heating regime, ${ }^{11}$ or an external resistor as in the case of a hot disk. ${ }^{12}$ These techniques can be applied for measuring thermal properties of different structures such as bulk, thick, and thin films. The main disadvantage of these techniques is the presence of thermal contact resistance between the heat source and the sample. Therefore, the use of such techniques for the study of samples with rough surfaces, like nanowires arrays, is very challenging.

Non-contact methods are based on the remote excitation of the thermal perturbation in the material. For instance, in photothermal methods, the thermal perturbation is induced by light irradiation. In this frame, there is no contact resistance, and this is the significant advantage of such methods, which does not require special pre-treatment of the studied sample. On the other hand, it is difficult to evaluate the amount of energy absorbed in the sample. Consequently, several issues can be expected when material heat capacity measurements are required.

In practice, methods with transient excitation of thermal perturbations allow the determination thermal diffusivity $(D)$, which is 
related to thermal conductivity $\left(\kappa_{s}\right)$ with the following equation:

$$
D=\frac{\kappa_{s}}{c \rho}
$$

where $c$ is the specific heat and $\rho$ is the density of the material. Therefore, to evaluate the thermal conductivity, both density and specific heat should be known, which is challenging for nanostructured materials.

Often, the density and specific heat for nanostructures are considered to be the same as for the bulk state. ${ }^{13,14}$ However, this assumption is crude, since changes in phonon dispersion and influence of phonon surface modes can affect the specific heat. ${ }^{15}$ Generally, features of the structure determine the application of the assumptions, ${ }^{16}$ while a universal approach applicable for all cases does not exist. Consequently, the development of new methods for the thermophysical property study of nanostructured materials remains an important issue.

In this paper, the study of thermal conductivity of nanostructured materials with the use of a photoacoustic technique in piezoelectric configuration is presented. ${ }^{17-22}$ An approach of simultaneous evaluation of thermal conductivity and heat capacity of material is proposed. ${ }^{23-25}$ Then, materials such as porous silicon samples (PS) and silicon nanowires arrays (SiNWs), fabricated by electrochemical and metal-assisted chemical etching (MACE) of a bulk silicon, are studied with the developed photoacoustic technique.

\section{EXPERIMENTAL DETAILS}

\section{A. Sample fabrication}

Porous silicon. The samples were fabricated by electrochemical etching of the p+-type monocrystalline $\mathrm{Si}$ wafer doped with boron with a resistivity about $0.01-0.02 \Omega \mathrm{cm}$. The thickness of the silicon wafer was $500 \mu \mathrm{m}$. The etching solution consists of hydrofluoric acid and ethanol [HF (49\%): $\left.\mathrm{C}_{2} \mathrm{H}_{5} \mathrm{OH}=1: 1\right]$. For obtaining porous silicon with porosity $P=45 \%, 55 \%$, and $65 \%$, different anodic current densities of $j=50 \mathrm{~mA} / \mathrm{cm}^{2}, 100 \mathrm{~mA} / \mathrm{cm}^{2}, 200 \mathrm{~mA} / \mathrm{cm}^{2}$, respectively, were applied. The etching time was set such as to obtain the same thickness of all fabricated porous layers, which is measured to be equal to $50 \mu \mathrm{m}$. The morphologies of the resulting porous silicon layers were observed via scanning electron microscopy (SEM). The SEM image of the prepared porous silicon sample with porosity $45 \%$ is shown in Fig. 1 .

Silicon nanowire arrays. Samples of silicon nanowires arrays were fabricated by the method of metal-assisted chemical etching (MACE) of a monocrystalline p-type silicon substrate doped with boron. The initial thickness of the silicon wafer was $300 \mu \mathrm{m}$ and resistance of initial wafer was $0.04-0.05 \Omega \mathrm{cm}$. Schematic illustration of the successive steps of metal-assisted chemical etching of silicon nanowires arrays is shown in Fig. 2(a).

The wafers were cleaned in an aqua solution of $5 \%$ hydrofluoric acid (HF) to remove the oxide layer from the substrates. After cleaning, substrates were immersed in a solution of $5 \mathrm{M}$ hydrofluoric acid and $0.02 \mathrm{M}$ silver nitrate. As a result, Ag nanoparticles were deposited onto the wafer surface. The next step is the formation of Si nanowires. For this purpose, the samples were immersed in an etching solution of $5 \mathrm{M}$ hydrofluoric acid and 30\% hydrogen

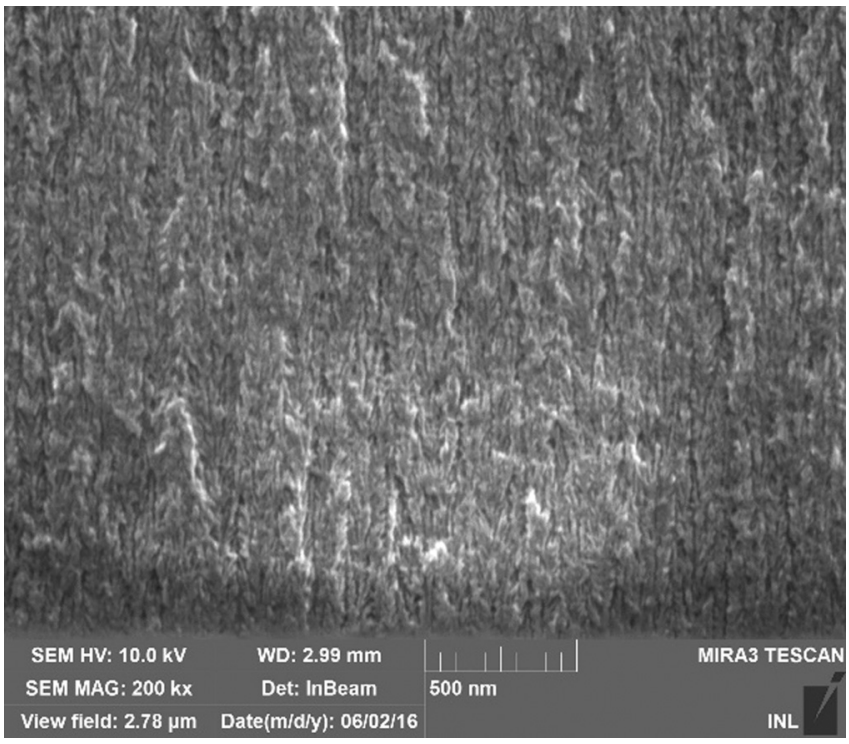

FIG. 1. Cross sectional SEM image of a porous silicon layer with porosity $P=45 \%$ fabricated by electrochemical etching.

peroxide. Then, to dissolve the Ag dendrites, the samples were immersed into $65 \% \mathrm{HNO}_{3}$. After that, it was rinsed with de-ionized water. Three series of the samples were fabricated with different etching times ( $35 \mathrm{~min}, 60 \mathrm{~min}$, and $90 \mathrm{~min}$ ), which lead to the formation of SiNWs with different thicknesses $(20 \mu \mathrm{m}, 35 \mu \mathrm{m}$, and $50 \mu \mathrm{m}$, respectively). The porosity of the fabricated SiNWs arrays is equal to $(55 \pm 5) \%$. The typical SEM image of a fabricated sample with the thickness of SiNW arrays equal to $50 \mu \mathrm{m}$ is shown in Fig. 2(b).

Porosity for all samples was first estimated with the gravimetric method followed by more precise control with the use of SEM images.

\section{B. Photoacoustic measurements}

Setup details. As mentioned above, we used the photoacoustic technique with piezoelectric recording. The piezoelectric detection principle has several advantages when compared to the more classical gas-microphone one, such as larger working frequency range. Additionally, it gives the possibility to study photothermal response directly from the sample. A schematic configuration of the photoacoustic measurement unit with a piezoelectric detection system is shown in Fig. 3. The experiments were carried out in the presence and absence of a covering liquid layer (water) with thickness equal to $1 \mathrm{~mm}$. The samples were irradiated by modulated laser radiation with a wavelength equal to $532 \mathrm{~nm}$ and the electric power of $2 \mathrm{~W}$. The square-wave modulation of the laser was performed by modulating its power source with the Tektronix AFG1022 generator. The parallel light beam was formed by the optical collimator system to form a spot on the sample surface with the size equal to $3 \mathrm{~mm}$. The absorption of the electromagnetic radiation induced the heating of the sample. As a result, thermoelastic stresses were 
(a)

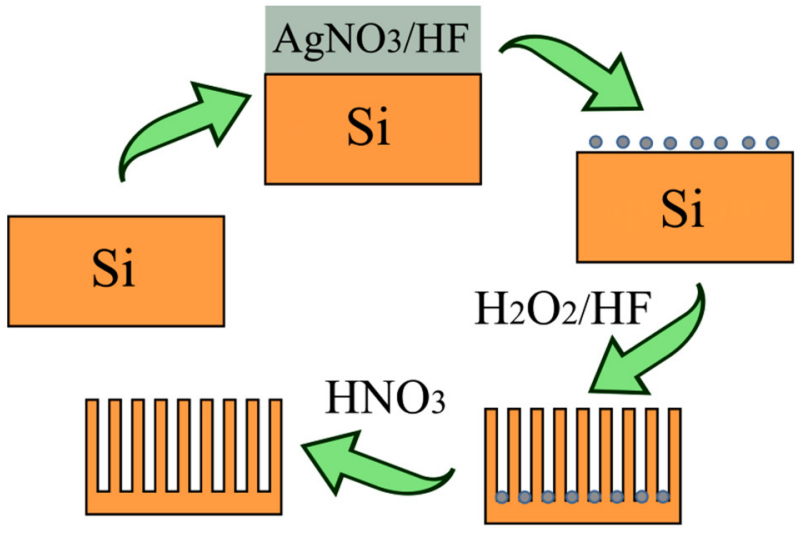

(b)

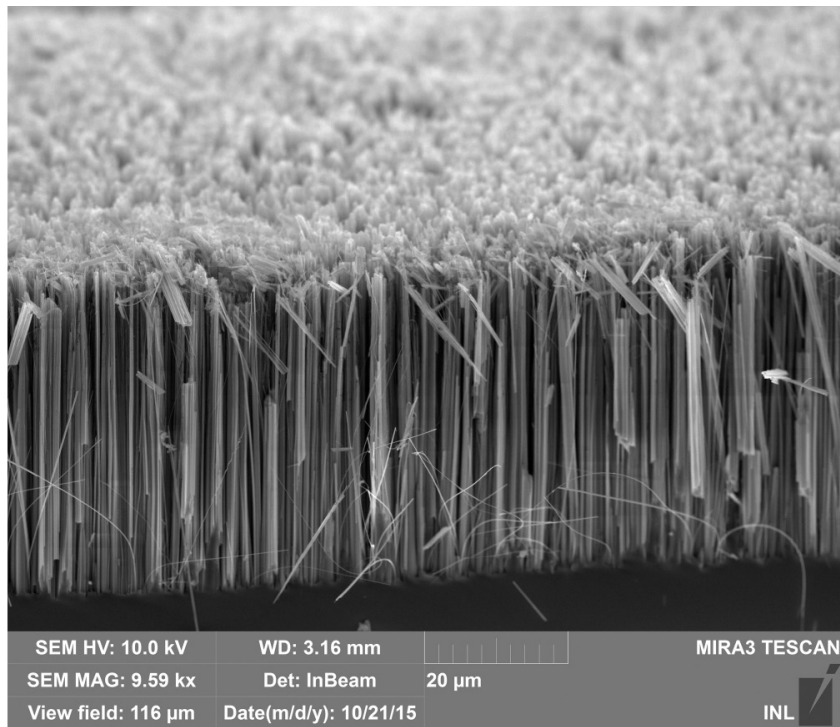

FIG. 2. The sketch of MACE process stages (a); SEM image of fabricated sample (b).

arising in the considered multilayered system. These stresses were detected by a piezoelectric transducer (PZT, PIC-151). A specific buffer layer with low thermal conductivity and thermal expansion coefficient was chosen for localization of thermoelastic sources in the studied sample and thus avoiding heat energy penetration into the piezoelectric transducer. This buffer was fabricated from the glass ceramic layer (thickness is equal to $1 \mathrm{~mm}$ ). The length and width of both the sample buffer layer and the PZT was $5 \times 5 \mathrm{~mm}^{2}$. Photoacoustic responses were recorded in the frequency range from $10 \mathrm{~Hz}$ to $20 \mathrm{kHz}$. The typical amplitude-frequency characteristics are presented in the Results section (see Fig. 4).

Analytic model. Let us consider the photoacoustic response formation in the multilayered structure shown in Fig. 3. The analysis of experimental amplitude-frequency characteristics was carried out in frames of quasi-stationary deformation assumptions. The spatial distribution of thermoelastic stresses that arise in the studied
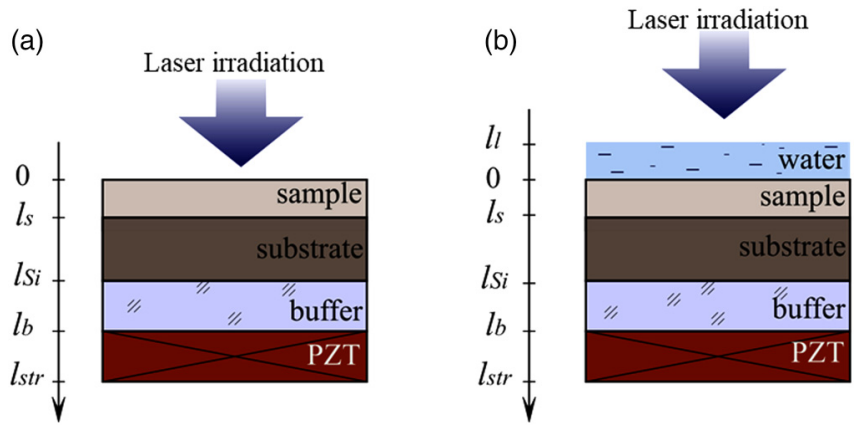

FIG. 3. Schematic sketch-view of a multilayered system in the case of absence (a) and presence (b) of the covering liquid layer. structure can be represented as follows: ${ }^{26}$

$$
\sigma(z)=\frac{E(z)}{1-v(z)} \int_{0}^{l_{s t r}} G(z, s) \sigma_{s}(s) d s
$$

here $E(z)$ and $v(z)$ are the spatial distributions of Young's modulus and Poisson's coefficient, $G(z)$ is Green's function, and $\sigma_{s}(z)$ is the thermoelastic stress source term. In the considered case, the source can be expressed as a thermoelastic force that depends on the temperature field in the layers,

$$
\sigma_{s}(z)=\frac{E(z)}{1-v(z)} \alpha_{T}(z) \theta(z, \omega)
$$

where $\alpha_{T}(z)$ is the thermal expansion coefficient of the material and $\theta(z, \omega)$ is the spatial distribution of variable component of temperature. The spatial distribution of the temperature in the multilayered structure can be evaluated by solving the heat conduction equation with the appropriate boundary conditions, ${ }^{27}$

$$
c \rho \frac{\partial \theta}{\partial t}=\frac{\partial}{\partial z}\left(\kappa(z) \frac{\partial \theta}{\partial z}\right)+I \alpha e^{-\alpha z}
$$

where $I$ is the intensity of the absorbed radiation and $\alpha$ is the optical absorption coefficient.

It should be noted that the effect of electron-hole recombination was neglected in the heat conduction equation, since the lifetime of carriers in porous silicon and silicon nanowires is small, and the recombination processes are fast enough in comparison with the period of heating. ${ }^{28,29}$

It should be noted that the effects of thermal interface resistance could be neglected because the ratio of the nano-Si sample 


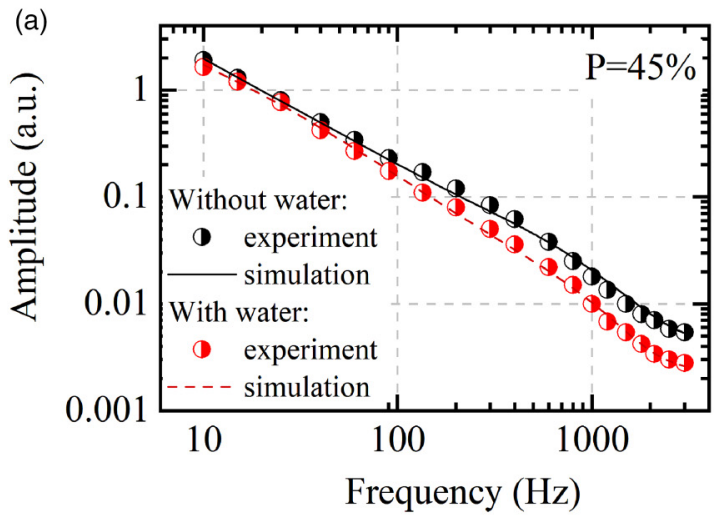

(c)

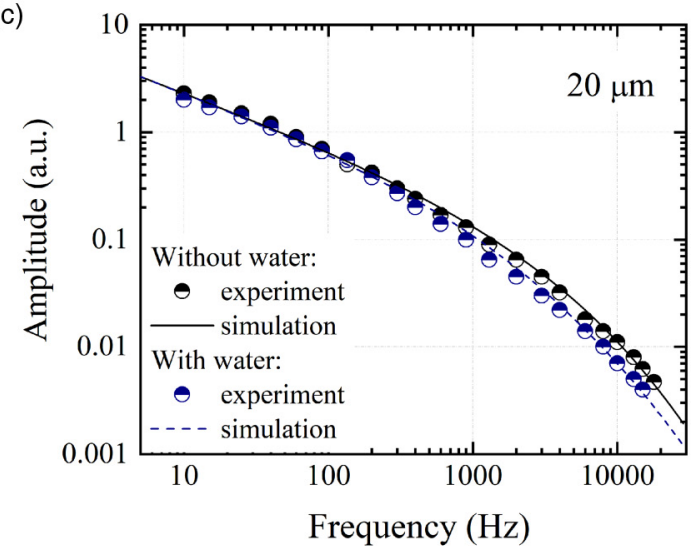

(b)

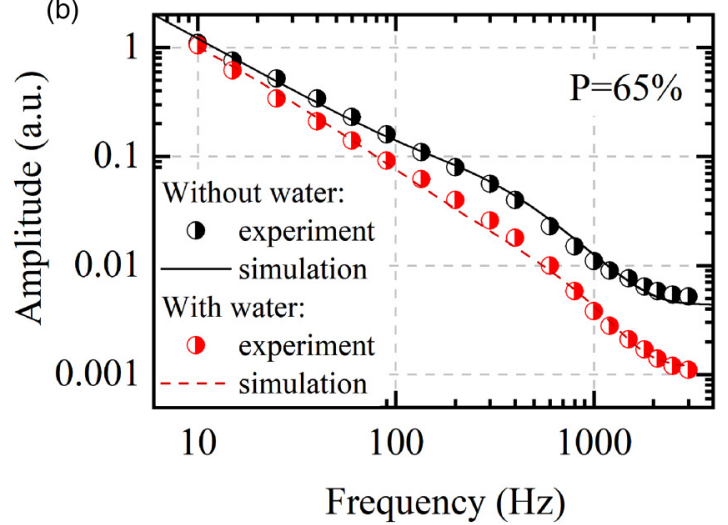

(d)

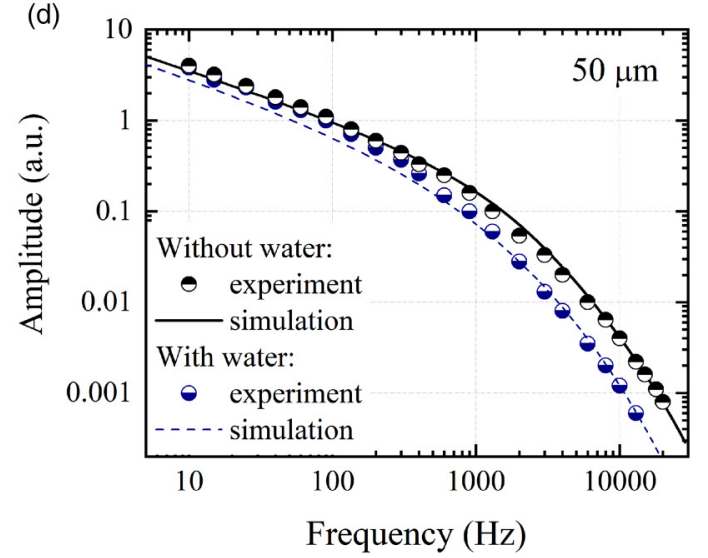

FIG. 4. The amplitude-frequency characteristics of the photoacoustic response of the studied system with a porous silicon layer [(a) and (b)] and silicon nanowires arrays [(c) and (d)] for the cases of absence and presence of a covering liquid layer.

thickness relative to its thermal conductivity $\left(\sim 10^{-5} \mathrm{~m}^{2} \mathrm{~K} / \mathrm{W}\right)$ was much higher for all series of samples than the typical thermal interface resistance value $\left(\sim 10^{-7} \mathrm{~m}^{2} \mathrm{~K} / \mathrm{W}\right) .^{30-32}$

Since, the voltage $U$ detected from piezoelectric transducer electrodes is proportional to the thermoelastic stresses inside the sample, ${ }^{33}$ photoacoustic response $[U(\omega)]$ depends on the temperature rise induced by the absorption of radiation. The correlation of the experimental and theoretical values of such a temperature rise allows the evaluation of the thermal diffusivity of the material D through inverse modeling.

\section{RESULTS AND DISCUSSION}

The typical amplitude-frequency characteristics of the photoacoustic response for the cases with and without the water covering layer are shown in Fig. 4. Photoacoustic characteristics for porous silicon samples with different porosities are presented in Figs. 4(a) and 4(b), while Figs. 4(c) and 4(d) stand for silicon nanowire arrays. In both materials, the amplitude dependencies with the frequency of photoacoustic signals change with porosity or thickness of layers. As we can see, there is a change of the curve slope on the amplitude-frequency variation for both series of samples. Such a behavior is related to the different behaviors of thermal and mechanical properties of the studied samples above the silicon substrate. The results of numerical simulations using the theoretical model described in Sec. II are plotted with continuous lines (3) in Fig. 4. Simulation results are in good agreement with the experimental data. The difference between photoacoustic formation mechanisms in porous silicon and silicon nanowires arrays analyzed in detail in Refs. 34.

Additional calorimetric measurements are required for evaluation of the heat capacity $C_{s}$ and the thermal conductivity $\kappa_{s}$ of nanostructured systems. For this purpose, the surface of the sample was covered with a liquid layer. As a result, the optical deposited energy is redistributed at the surface of the structure. The heat fluxes at the interface "nanostructured solid/fluid" should be equal in accordance with the Fourier law:

$$
\left.\kappa_{l} \frac{\partial T}{\partial z}\right|_{z=0-0}=\left.\kappa_{s} \frac{\partial T}{\partial z}\right|_{z=0+0},
$$

where $\kappa_{l}$ and $\kappa_{s}$ are the thermal conductivities of the liquid and the sample, respectively. 
(a)

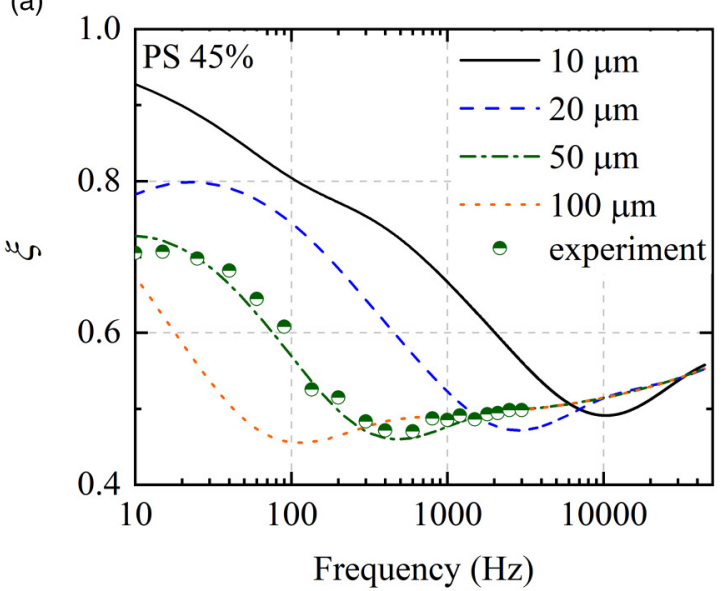

(c)

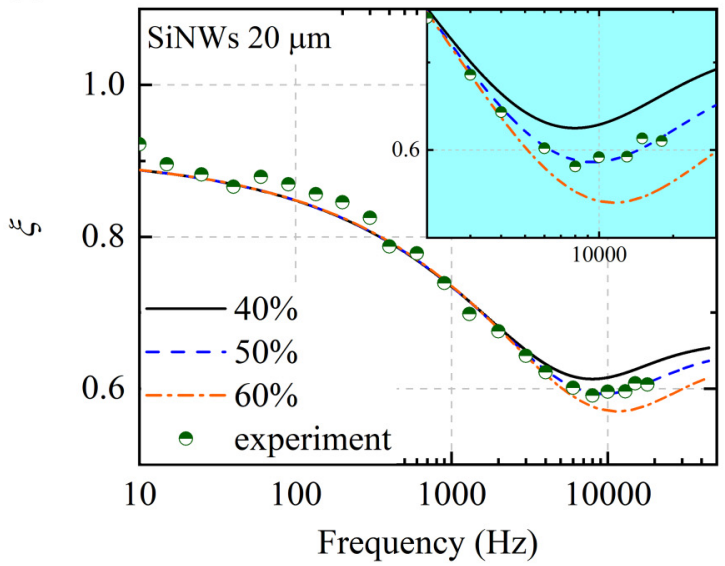

(b)

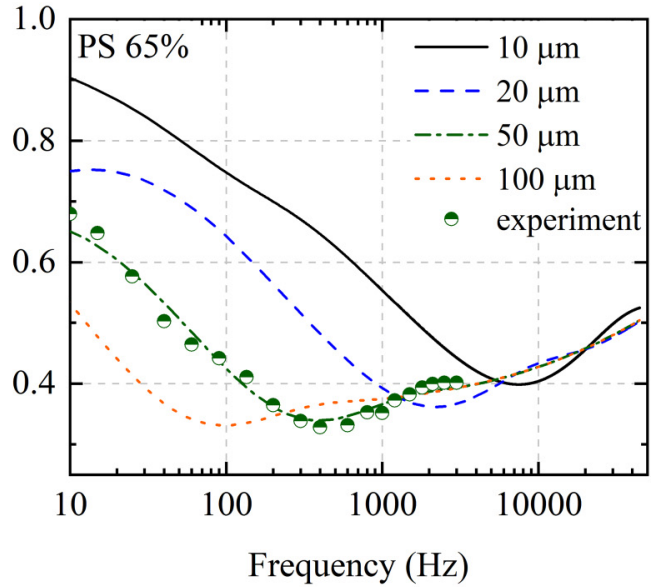

(d)

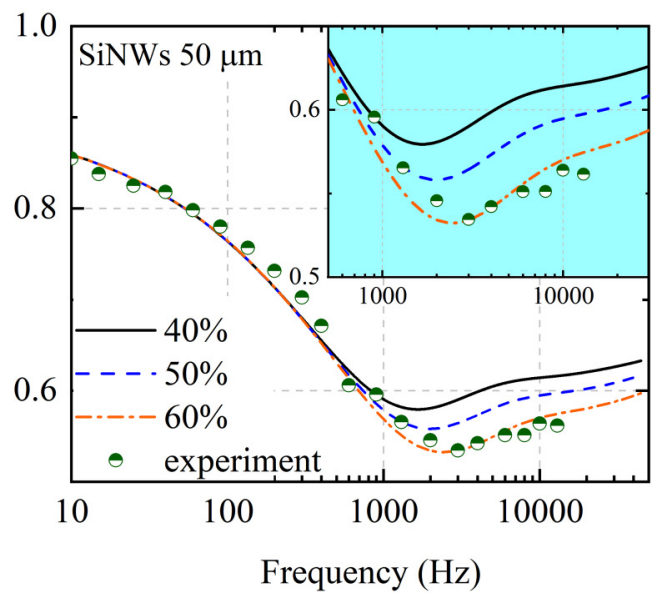

FIG. 5. Experimental (dots) and simulated (lines) frequency dependencies of the amplitude ratio measured for the sampled covered by liquid and the initial one. The simulated curves are presented for different thicknesses of the porous silicon with porosities $45 \%$ (a) and $65 \%$ (b), and for different porosities of silicon nanowires arrays with thicknesses equal to $20 \mu \mathrm{m}$ (c) and $50 \mu \mathrm{m}$ (d).

In the case of the dry sample, all the thermal energy is concentrated inside the porous silicon or silicon nanowire layer. In the case with liquid film, energy was redistributed between the liquid and the solid in contact. The ratio of thermal energy at the interface is determined by the correlation of the thermal conductivities of the contact media. Furthermore, the amount of thermal energy that penetrates inside the sample depends on the thermal conductivity of the studied material. As it was mentioned above, the parameters of the photoacoustic response depend on the source of thermoelastic stresses, which will be modified due to the heat outflow into the liquid layer.

The typical amplitude-frequency characteristics of the photoacoustic response in the cases of absence (1) and presence (2) of the surface liquid layer are shown in Fig. 4. In the presence of such a layer, the amplitude of the photoacoustic response systematically decreases (2) for both series of the studied samples and the reduction is more important in increasing frequency.
It should be noted that such a decrease in the amplitude depends on the thickness of the sample. In the case of thin layers of PS or SiNWs, the heat perturbation effectively penetrates in the substrate with a high value of thermal conductivity $\left(\kappa_{\mathrm{Si}} \ll \kappa_{s}, \kappa_{\mathrm{Si}} \gg \kappa_{l}\right)$. Thus, influence of the interface between nanostructured solid and fluid is not so crucial, especially at the low frequency range. With increasing

TABLE I. Thermophysical parameters of the porous silicon samples with different porosities.

\begin{tabular}{lcccc}
\hline \hline No. & $P(\%)$ & $C_{s}\left(\mathrm{~kJ} / \mathrm{K} \mathrm{m}^{3}\right)$ & $\begin{array}{c}\text { Experiment } \kappa_{s} \\
(\mathrm{~W} / \mathrm{m} \mathrm{K})\end{array}$ & $\begin{array}{c}\text { Literature data } \\
\kappa(\mathrm{W} / \mathrm{m} \mathrm{K})\end{array}$ \\
\hline 1 & $45 \pm 5$ & $871 \pm 79$ & $2.4 \pm 0.3$ & $2.8^{37}$ \\
2 & $55 \pm 7$ & $713 \pm 110$ & $1.9 \pm 0.3$ & $1.4^{37}$ \\
3 & $65 \pm 6$ & $555 \pm 95$ & $1.3 \pm 0.3$ & $1.0^{37}$ \\
\hline \hline
\end{tabular}


TABLE II. Thermophysical parameters of the silicon nanowires arrays with different thicknesses.

\begin{tabular}{lcccc}
\hline \hline No. & $l_{s}(\mu \mathrm{m})$ & $C_{s}\left(\mathrm{~kJ} / \mathrm{K} \mathrm{m}^{3}\right)$ & $\begin{array}{c}\text { Experiment } \kappa_{s} \\
(\mathrm{~W} / \mathrm{m} \mathrm{K})\end{array}$ & $\begin{array}{c}\text { Literature data } \\
\kappa(\mathrm{W} / \mathrm{m} \mathrm{K})\end{array}$ \\
\hline 1 & 20 & $792 \pm 63$ & $7.1 \pm 0.4$ & $8^{38} ; 5.8^{39}$ \\
2 & 35 & $745 \pm 76$ & $6.3 \pm 0.4$ & $4.2^{39}$ \\
3 & 50 & $670 \pm 79$ & $5.7 \pm 0.4$ & $5.4^{38}$ \\
\hline \hline
\end{tabular}

frequency, the length of the thermal wave decreases $(\lambda=\sqrt{a / 2 \omega}){ }^{35}$ and heat perturbation in this case is localized in PS or SiNWs.

Therefore, as one can see in Fig. 4, the amplitudes of photoacoustic signals in the cases of absence and occurrence of the water layer are approximately the same for frequencies $<a l_{s}^{2}$. With increasing frequency, when the length of the thermal wave becomes commensurate to the sample thickness, the thermal energy is more efficiently released from the silicon nanostructures inside the liquid.

Dependencies of the ratio of frequency amplitudes " $\xi$ " (see Fig. 5) for the wet sample and the pristine one are more informative. In this figure, one can see that the magnitude of the PA signal decrease is due to the presence of the covering liquid film. The decrease of the magnitude has a well-pronounced minimum. The position of the minimum depends on the thickness of the nanostructured layer as well as the thermal physical properties. Figure 3 shows quite a good agreement between simulations and experimental results. It is also necessary to mention at this point that experimental points for a thinner sample of SiNWs arrays are closer to the simulated curve corresponding to the porosity of $50 \%$, while the points for a thickest sample are closer to the simulated curve corresponding to the porosity of $60 \%$. The latter corresponds well with the above-mentioned assumption that during fabrication of SiNWs, porosity slightly increases.

Correlations of two cases, defined by the presence and the absence of the covering liquid layer gives us the possibility for simultaneous evaluation of the values of the heat capacity $C_{s}$ and of the thermal conductivity $\kappa_{s}$. Such evaluation was performed by the fitting of experimental amplitude-frequency characteristics with simulated ones. The extracted and literature values are shown in Table I for porous silicon samples and in Table II for silicon nanowires arrays. Additionally, obtained results are presented in Fig. 6.

Thermal conductivity of the porous silicon samples decreases with porosity according to the following expression: $\kappa_{s}=(1-P)^{3} \kappa_{\mathrm{Si}} \cdot{ }^{36}$ As one can see, the heat capacity also decreases with increasing porosity of the layer. The values of heat capacities obtained using the photoacoustic approach agree with those that can be estimated from the approximation of the effective medium: $\rho_{s} C_{s}=(1-P) \rho_{\mathrm{Si}} C_{\mathrm{Si}}$.

The heat capacity of the nanowire arrays decreases with increasing layer thickness. However, owing to the average dimensions of the nanowire diameter $(\sim 100 \mathrm{~nm})$, it can be assumed that the value of the specific heat is the same as the specific heat of crystalline silicon. ${ }^{40}$ Consequently, the change in the heat capacity can be related to the variation of the porosity of the etched nanowires as it was observed in Fig. 5.

As can be seen from Table II, the thermal conductivity value decreases with increasing SiNW layer thickness. Such a decrease can be explained by increasing the structure heterogeneity, broken nanowires, and porosity arising during the etching process. ${ }^{41}$ This consideration is correlated with the previous assumption regarding the reduction in heat capacity with the increase in the thickness of the layer.

\section{CONCLUSIONS}

An experimental method based on the heat flux redistributions on the surface of the studied structures is proposed in order to appraise material heat capacity and thermal conductivity simultaneously. This method is based on the deposition of covering a liquid layer on the sample surface, which acts like a heat sink and leads to the redistribution of heat fluxes. In this framework, the photoacoustic technique with piezoelectric recording has been chosen to achieve nondestructive contactless thermal properties diagnostics. The features of the amplitude-frequency characteristics
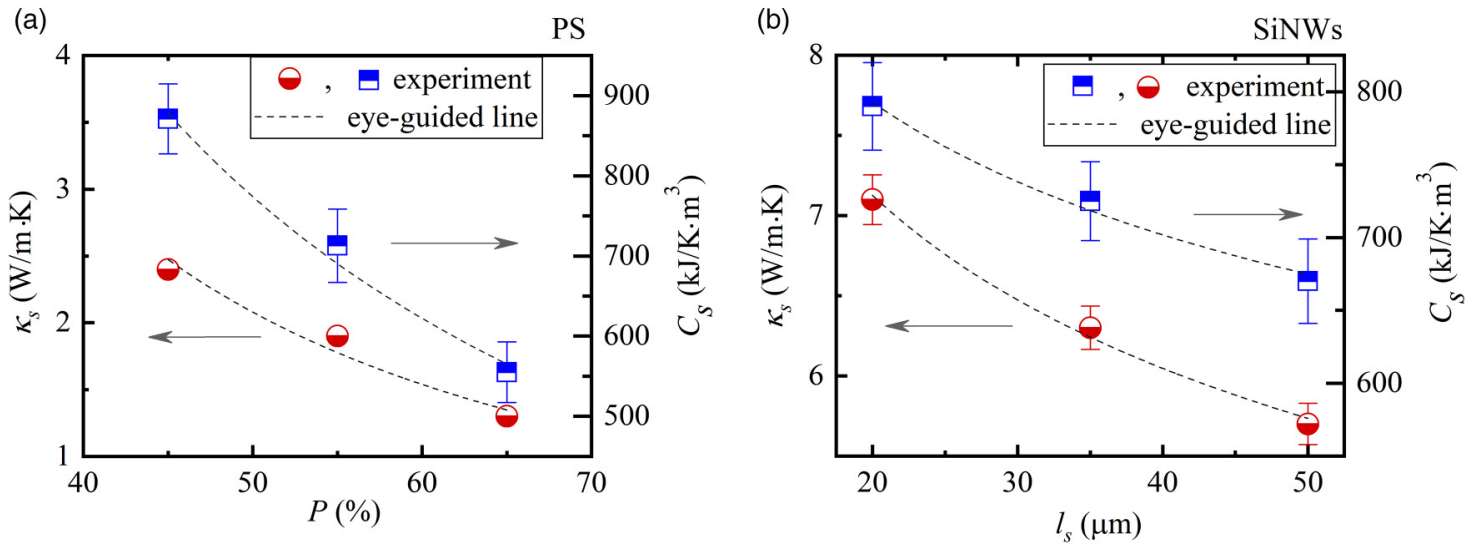

FIG. 6. Thermophysical parameters of the porous silicon samples with different porosities (a) and the silicon nanowires arrays with different thicknesses (b). 
of the photoacoustic response for silicon-based nanostructured materials with different morphologies are analyzed with and without a covering liquid film.

First, the approach was tested on the samples of porous silicon with different porosities. The porous silicon was chosen because of the possibility to precisely control the thickness of the sample and its porosity during the fabrication processes. The experimental results are in good agreement with the proposed theoretical model of the photoacoustic response formation in such structures.

Then, the methodology was applied for the silicon nanowire arrays fabricated by metal-assisted chemical etching. It was found that thermal conductivity and the heat capacity of the samples decreased with the increasing etching time. Considering the average sizes of nanowire diameters, one can assume that the specific heat capacity of SiNW arrays is the same as the one of crystalline silicon. Therefore, such a behavior of the heat capacity could be associated with the increase in porosity of the studied materials as it was observed on the amplitude frequency dependencies. The decrease of the thermal conductivity could be also explained by the increase of the porosity and the growth of the structure heterogeneities occurred during the silicon substrate etching.

\section{SUPPLEMENTARY MATERIAL}

See the supplementary material containing SEM images of all samples used in the work.

\section{ACKNOWLEDGMENTS}

This publication contains the results obtained in the frames of research work "Features of photothermal and photoacoustic processes in low-dimensional silicon-based semiconductor systems" (Ministry of Education and Science of Ukraine, State Registration No. 0118U000242) and project HotLine (Agence Nationale de la Recherche, France, Project No. ANR-19-CE09-0003). This work was partially funded by the CNRS Energy Unit (PEPS Cellule ENERGIE 2019) through the project ImHESurNaASA. Mykola Isaiev and David Lacroix would also like to acknowledge the partial financial support of the scientific pole EMPP of the University of Lorraine.

\section{DATA AVAILABILITY}

The data that support the findings of this study are available from the corresponding author upon reasonable request.

\section{REFERENCES}

'Y. V. Ryabchikov, V. Lysenko, and T. Nychyporuk, J. Phys. Chem. C 118, 12515 (2014).

${ }^{2}$ A. Al-Kattan, Y. V. Ryabchikov, T. Baati, V. Chirvony, J. F. Sánchez-Royo, M. Sentis, D. Braguer, V. Y. Timoshenko, M.-A. Estève, and A. V. Kabashin, J. Mater. Chem. B 4, 7852 (2016).

${ }^{3}$ O. Olikh and K. Voytenko, Ultrasonics 66, 1 (2016).

${ }^{4}$ C. F. Ramirez-Gutierrez, J. D. Castaño-Yepes, and M. E. Rodriguez-Garcia, Optik 173, 271 (2018).

${ }^{5}$ C. F. Ramirez-Gutierrez, A. Medina-Herrera, L. Tirado-Mejía, L. F. Zubieta-Otero, O. Auciello, and M. E. Rodriguez-Garcia, J. Lumin. 201, 11 (2018).

${ }^{6}$ D. G. Cahill, K. Goodson, and A. Majumdar, J. Heat Transfer 124, 223 (2002).

${ }^{7}$ S. Gomès, A. Assy, and P. O. Chapuis, Phys. Status Solidi A 212, 477 (2015).

${ }^{8}$ D. G. Cahill, Rev. Sci. Instrum. 61, 802 (1990).
${ }^{9} \mathrm{~L} . \mathrm{Lu}, \mathrm{W}$. Yi, and D. Zhang, Rev. Sci. Instrum. 72, 2996 (2001).

${ }^{10}$ F. Völklein, Thin Solid Films 188, 27 (1990).

${ }^{11}$ M. Okuda and S. Ohkubo, Thin Solid Films 213, 176 (1992).

${ }^{12}$ M. Gustavsson, E. Karawacki, and S. E. Gustafsson, Rev. Sci. Instrum. 65, 3856 (1994).

${ }^{13}$ Thermal Conductivity-Theory, Properties, and Applications, edited by T. M. Tritt (Kluwer Academic Plenum Publishers, New York, 2004).

${ }^{14}$ C. Chiritescu, D. G. Cahill, N. Nguyen, D. Johnson, A. Bodapati, P. Keblinski, and P. Zschack, Science 315, 351 (2007).

${ }^{15} \mathrm{G}$. Chen, J. Nanoparticle Res. 2, 199 (2000).

${ }^{16}$ K. Termentzidis, Nanostructured Semiconductors: Amorphization and Thermal Properties (Pan Stanford, 2017).

${ }^{17}$ P. Lishchuk, D. Andrusenko, M. Isaiev, V. Lysenko, and R. Burbelo, Int. J. Thermophys. 36, 2428 (2015).

${ }^{18}$ R. Burbelo, D. Andrusenko, M. Isaiev, and A. Kuzmich, Arch. Metall. Mater. 56, 1157 (2011).

19. A. Balderas-López, Rev. Sci. Instrum. 77, 064902 (2006).

${ }^{20}$ K. G. Biswas, T. D. Sands, B. A. Cola, and X. Xu, Appl. Phys. Lett. 94, 223116 (2009).

${ }^{21}$ B. Abad, M. Rull-Bravo, S. L. Hodson, X. Xu, and M. Martin-Gonzalez, Electrochim. Acta 169, 37 (2015).

${ }^{22}$ Z. Šoškić, S. Ćirić-Kostić, and S. Galović, Int. J. Therm. Sci. 109, 217 (2016).

${ }^{23}$ S. Alekseev, D. Andrusenko, R. Burbelo, M. Isaiev, and A. Kuzmich, J. Phys. Conf. Ser. 278, 012003 (2011).

${ }^{24}$ M. Isaiev, K. Voitenko, V. Doroshchuk, D. Andrusenko, A. Kuzmich, A. Skryshevskii, V. Lysenko, and R. Burbelo, Phys. Procedia 70, 586 (2015).

${ }^{25}$ D. Andrusenko, M. Isaiev, A. Kuzmich, V. Lysenko, and R. Burbelo, Nanoscale Res. Lett. 7, 411 (2012)

${ }^{26} \mathrm{M}$. Isaiev, D. Andrusenko, A. Tytarenko, A. Kuzmich, V. Lysenko, and R. Burbelo, Int. J. Thermophys. 35, 2341 (2014).

${ }^{27}$ K. Voitenko, D. Andrusenko, A. Pastushenko, M. Isaiev, A. G. Kuzmich, and R. M. Burbelo, J. Nano Electron. Phys. 9, 04021 (2017).

${ }^{28}$ D. K. Markushev, D. D. Markushev, S. Aleksić, D. S. Pantić, S. Galović, D. M. Todorović, and J. Ordonez-Miranda, J. Appl. Phys. 126, 185102 (2019).

${ }^{29}$ D. D. Markushev, J. Ordonez-Miranda, M. D. Rabasović, S. Galović, D. M. Todorović, and S. E. Bialkowski, J. Appl. Phys. 117, 245309 (2015).

${ }^{30}$ E. T. Swartz and R. O. Pohl, Rev. Mod. Phys. 61, 605 (1989).

${ }^{31}$ B. Deng, A. V. Chernatynskiy, M. Khafizov, D. H. Hurley, and S. R. Phillpot, J. Appl. Phys. 115, 084910 (2014).

${ }^{32} \mathrm{H}$. Wang, Y. Xu, M. Shimono, Y. Tanaka, and M. Yamazaki, Mater. Trans. 48, 2349 (2007).

${ }^{33}$ K. Dubyk, L. Chepela, P. Lishchuk, A. Belarouci, D. Lacroix, and M. Isaiev, Appl. Phys. Lett. 115, 021902 (2019).

${ }^{34} \mathrm{~K}$. Dubyk, A. Pastushenko, T. Nychyporuk, R. Burbelo, M. Isaiev, and V. Lysenko, J. Phys. Chem. Solids 126, 267 (2019).

${ }^{35}$ H. S. Carslaw and J. C. Jaeger, Conduction of Heat in Solids, 2nd ed. (Clarendor Press, Oxford, 1959).

${ }^{36}$ P. J. Newby, in Porous Silicon: From Formation to Application, edited by G. Korotcenkov (CRC Press, 2016), Vol. 1, pp. 237-250.

${ }^{37}$ V. Lysenko, L. Boarino, M. Bertola, B. Remaki, A. Dittmar, G. Amato, and D. Barbier, Microelectron. J. 30, 1141 (1999).

${ }^{38}$ A. I. Hochbaum, R. Chen, R. D. Delgado, W. Liang, E. C. Garnett, M. Najarian, A. Majumdar, and P. Yang, Nature 451, 163 (2008).

${ }^{39} \mathrm{M}$. Isaiev, O. Didukh, T. Nychyporuk, V. Timoshenko, and V. Lysenko, Appl. Phys. Lett. 110, 011908 (2017).

${ }^{40}$ A. A. R. A. I. Gusev, Nanocrystalline Materials, 1st ed. (Cambridge International Science Publishing Ltd., Cambridge, 2004), pp. 177-190.

${ }^{41}$ S. P. Rodichkina, L. A. Osminkina, M. Isaiev, A. V. Pavlikov, A. V. Zoteev, V. A. Georgobiani, K. A. Gonchar, A. N. Vasiliev, and V. Y. Timoshenko, Appl. Phys. B 121, 337 (2015). 